\title{
23
}

\section{A Simulation Study on Using Inverse Transient Analysis for Leak Detection in Water Distribution Networks}

\author{
Bryan Karney, David Khani, Mahmoud R. Halfawy and \\ Osama Hunaidi
}

Inverse transient analysis, developed by several researchers in recent years as a promising and low-cost leak detection technique, has been successfully demonstrated under laboratory settings. However, the feasibility and technical limitations of this technique under actual field conditions has yet to be definitely established. This chapter reports on the initial simulation phase of a study that aims to assess the applicability and effectiveness of inverse transient analysis in the detection of leaks in real water distribution systems. Simulation was conducted and field tests are planned for a selected area of the City of Regina's municipal water distribution network. Scenarios of various transient severity and leak sizes were simulated. Insight gained from the simulation provided a preliminary assessment of the effectiveness and feasibility of using the inverse transient analysis method for leak detection.

\subsection{Introduction}

Leaks from water distribution systems are a major concern to water utilities for several reasons, including loss of limited water resources and pumping energy, economic loss, and increased health risks due to escalating breakage

Karney, B., D. Khani, M. Halfawy and O. Hunaidi. 2009. "A Simulation Study on Using Inverse Transient Analysis for Leak Detection in Water Distribution Networks." Journal of Water Management Modeling R235-23. doi: 10.14796/JWMM.R235-23.

(C) CHI 2009 www.chijournal.org ISSN: 2292-6062 (Formerly in Conceptual Modeling of Urban Water Systems. ISBN: 978-0-9808853-2-3) 
rates and the possibility of intrusion by contaminants. Devising efficient and economic leak detection and management strategies is becoming a high priority for almost all water utilities worldwide. Although many new methods for leak detection have recently been developed and deployed, questions about their efficiency, effectiveness and economic viability have impeded their wide scale deployment. Consequently, many water utilities still do not have an effective strategy for leak detection and management.

Water utilities typically employ a wide range of leak detection methods, but acoustic methods are the most commonly used. These methods generally involve attaching listening devices (e.g. listening rods, aqua-phones) to the pipes or appurtenances (e.g. fire hydrants, valves) to detect leak-induced sound. Ground microphones can also be used to listen and pinpoint leaks by listening on the pavement surface or soil directly above the pipe. Modern listening devices use signal amplifiers and noise filters to enhance the signal quality. Alternatively, noise loggers may be permanently or temporarily deployed in groups to survey large areas for leaks. More recently, leak noise correlators are being used to accurately pinpoint leak locations. The correlators work by recording sound signals at two points bracketing the location of a suspected leak using vibration sensors (e.g. accelerometers or hydrophones) and correlating the two signals to detect the presence of a leak and to accurately pinpoint its location. The effectiveness of acoustic leak detection methods has been successfully demonstrated for metallic pipes (Fantozzi et al. 1993, Fuchs and Riehle 1991) and plastic pipes (Hunaidi et al. 2000). A detailed review of acoustic leak detection methods is presented by Hunaidi et al. (2004).

Another leak detection method involves dividing the distribution system into well-defined district metering areas (DMAs). Each DMA comprises between 500 and 3000 properties that are supplied through a single pipe, where a flow meter is installed. Leakage level in a DMA is determined based on the minimum night flow minus the legitimate night demand and estimated unavoidable background leakage. DMAs help identify areas of the pipe network that suffer from excessive leakage, which can then be targeted for acoustic leak detection and pinpointing surveys. Guidelines for setup, maintenance, and monitoring of DMAs are presented by IWA (2007).

Several other non-acoustic leak detection technologies have been developed and tested, though with limited success. Examples of these technologies include tracer gas methods, liquid sensing cables, infrared thermography, and ground-penetrating radar. Although many of these methods proved effective in a number of cases, no definite answer regarding their effectiveness could be reliably established (Hunaidi et al. 1999). 
In selecting the most appropriate leak detection methods for a particular utility or scenario, a number of economical, operational, environmental, social, and legal factors should be considered (Hunaidi 2005). Various leak detection methods typically have different limitations, capabilities, costs, and benefits. The specific characteristics of the pipelines (e.g. age, leakage and break frequency, etc.) and the utility operations (e.g. cost of water, availability of trained and skilled staff, pressure management strategies, leak detection budget, water quality concerns, renewal strategies, etc.), along with other social and environmental factors typically determine a method's local applicability and feasibility.

An alternative leak detection technique involves pressure measurements and the inverse analysis (or back calculation) of the system hydraulic characteristics to determine the presence of leaks. Besides its apparent low cost and non-intrusive nature, this technique has the potential to detect and locate leaks at greater distances from the leak site, a clear advantage over the acoustic techniques. This benefit arises from the greater energy and superior propagation distances of a lower-frequency transient wave over an acoustic leak signal. These characteristics also make the propagation of pressure transients less sensitive to pipe material (i.e., plastics vs. metal), diameter, and sensor-to-sensor spacing. Also, unlike DMA-based leakage monitoring strategies, the inverse transient analysis approach would be able to pinpoint leaks or locate leaks within much smaller areas.

This chapter reports on the initial simulation phase of a study that aims to assess the applicability and effectiveness of inverse transient analysis to the detection of leaks in real water distribution systems. Simulation was conducted and field tests are planned for a specifically selected area of the City of Regina's municipal water distribution pipe network. Scenarios for various transient severity and leak size were simulated to analyze the relationship between transient severity and leak sizes, and to prepare proper field procedures and guidelines. Based on the insight gained from the simulation, a preliminary assessment on the effectiveness and feasibility of using the inverse transient method for leak detection is made.

\subsection{Inverse Transients for Leak Detection}

Since its formal introduction in 1994, inverse transient analysis (ITA) has been used by several researchers as a promising leak detection technique, having well demonstrated its power in laboratory and numerical settings. This method constitutes a low-cost and easy-to-implement alternative to the 
more costly and time-consuming leak detection techniques. However, its feasibility has yet to be fully established in actual field conditions. In addition to the difficulty of obtaining reliable and calibrated transient models, some systems have understandable concerns from the possible risks of introducing even controlled transients into the distribution system.

ITA involves injecting hydraulic transients with known intensity at given locations in the network (e.g. via controlled opening/closing of a fire hydrant). The transient signals are then recorded at various predetermined locations throughout the system. In parallel, a computer model of the network is coded into a transient analysis software program in which essentially identical transient events are introduced. The model is run for numerous sets of system parameters, a process facilitated by optimization routines such as genetic algorithms, where the objective function seeks to minimize the sum of squared differences between measured and computed pressure responses, until the best match between the measured and the computer generated responses is obtained. Discrepancies between response signatures (pressure traces at certain locations) can indicate the presence of leaks, assuming that accurate information regarding system condition and demands is available.

The study by Liggett and Chen (1994) was the first that proposed using ITA for leak detection. An earlier study by Pudar and Liggett (1992) introduced the idea of performing inverse analysis for leak detection under steady state conditions. That study concluded that steady state inverse analysis is unlikely to provide definitive leak detection results primarily due to the low number of data points since each steady state simulation only yields a single data point at each measurement station, which suggested the more data-rich transient approach.

The ITA method for leak detection proposed by Liggett and Chen (1994) was refined and tested by several subsequent studies. These studies mainly introduced new techniques to extend and improve optimization methods, sensor placement, and improvements in transient modeling. Moreover, the application of the ITA method has become more feasible and efficient with the use of more advanced pressure loggers and computer systems. Colombo et al. (2008) presented a review of transient-based leak detection approaches.

The use of ITA for leak detection has been tested in numerous studies reported in the literature. Besides the many numerical case studies and computer simulations reported (e.g. Vitkovsky et al. 2000), several studies validated the method through laboratory experiments (e.g. Covas et al. 2001 and Tang et al. 2001), and to some extent, in field trials (e.g. Saldarriaga et al. 2006). In general, the inverse transient approach performed rather well at 
pinpointing leaks in carefully controlled laboratory settings. However, significant work is still needed to validate the method in actual field conditions, and to further develop it into a practical and effective leak detection method.

A known limitation of the ITA method is its reliance on the availability of an accurate transient model of the network. Collecting accurate system parameters (e.g. roughness, demand, valve positions, etc.) can sometimes be impractical or expensive. To overcome this limitation, several researchers have devised alternative approaches that do not require accurate predictions of the transient signal, and therefore do not solely rely on the accuracy of the system's parameters. Examples of these approaches include the frequency domain analysis of the system's response, or the analysis of reflected transient signals. In a real water distribution network, especially when accurate data about the system is not available, combining the use of the ITA method with these methods will be a more practical and effective approach.

The promise and potential of the ITA method, coupled with the accumulated body of knowledge and the theoretical insights gained from previous work, the reasonable successes demonstrated by a range of lab and field experiments, and the pressure to implement cost-effective leak detection methods, have created a strong desire to validate the ITA method in the field and to further develop it into a practical solution in the near future.

\subsection{Background of the Simulation Study}

A research project was launched to assess the feasibility and technical limitations of the ITA method in water distribution systems, and to further develop operational guidelines for its application. In this project, an area in the City of Regina has been selected for the field-testing. This area is used as a test DMA as part of another research project, where the night supply and consumption have been accurately monitored. The area was surveyed for leaks using acoustic methods, and all leaks were repaired. The planned field work involves simulating leaks at a number of fire hydrants, and applying the inverse transient analysis method to detect and pinpoint the simulated leaks.

A simulation study was conducted in preparation for the field-testing. Several simulation scenarios were studied to analyze the main factors affecting the effectiveness and feasibility of the ITA method (e.g. leak size, transient severity, number of pressure transducers, effects of multiple leaks, 
etc.). In particular, two main and intensely practical questions were emphasized: (i) to what extent does the success of the method depend on the severity of the transients?; and (ii) how is the smallest detectable leak size related to the severity of the transient event?

The test area comprises 250 properties with $2693 \mathrm{~m}$ of asbestos cement (AC) pipes of diameter between 150 and $250 \mathrm{~mm}$. A skeletonized EPANET representation of the network is presented in Figure 23.1. Based on the hydraulic model, the average pressure in the area is about $400 \mathrm{kPa}$. Due to concerns by the City staff over the potential risks of inducing transients into the system, a decision was made to impose pressure restrictions, which could possibly limit the effectiveness of the ITA testing. The limits demanded by the city gave rise to a minimum/maximum pressure envelope of 14 to $50 \mathrm{~m}$, within which the tests can hopefully produce viable results. 


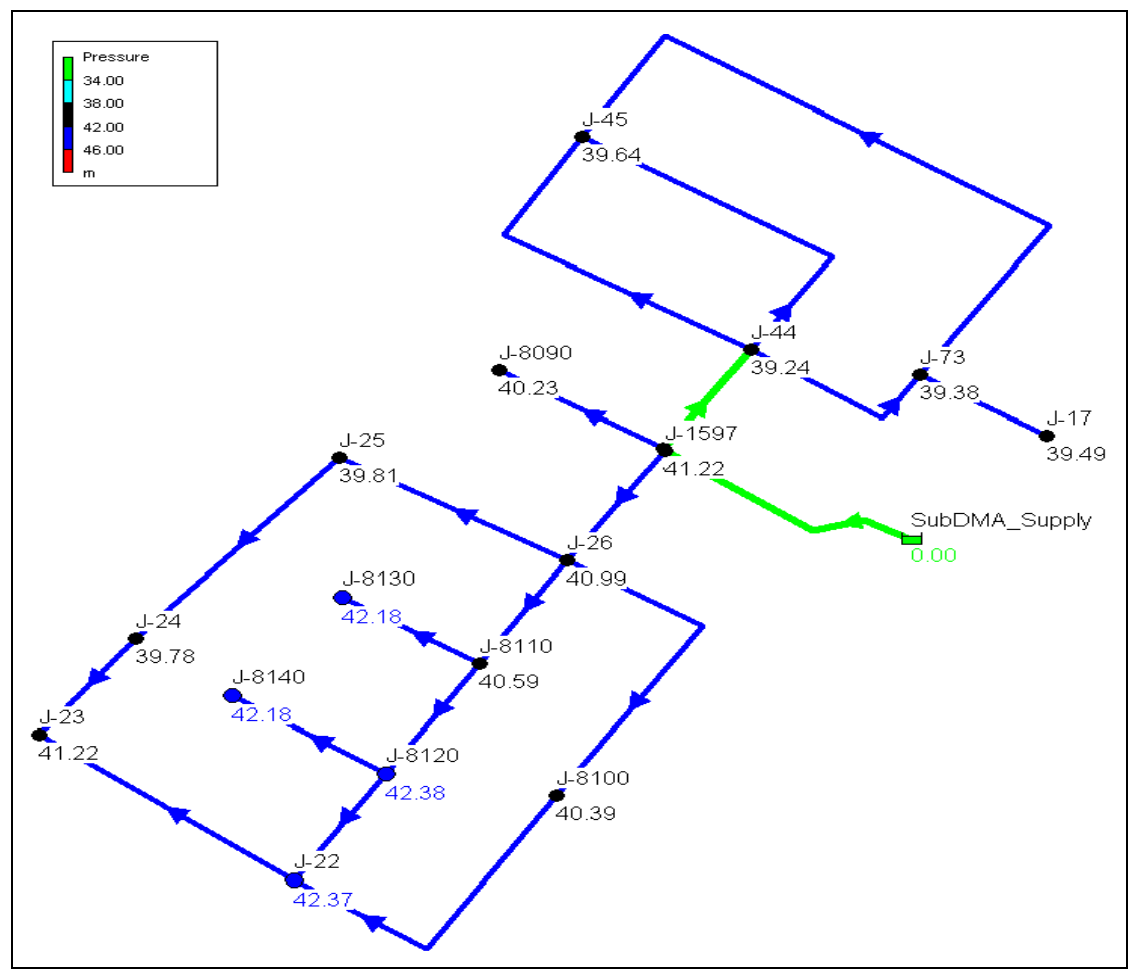

Figure 23.1 Skeletonized layout of the test area. Steady state pressures $(\mathrm{m})$ for a $50 \mathrm{~L} / \mathrm{s}$ hydrant discharge at node $\mathrm{J}-44(57 \mathrm{psi}=39 \mathrm{~m})$, are indicated at designated nodes.

\subsection{Methodology}

\subsubsection{Creating the Transients}

Transients are to be produced at one point in the network by opening and closing, in controlled fashion, a fire hydrant situated at node J-44 (Figure 23.1). A simple device consisting of a hose fitted with an electronically actuated flow control valve can be fitted to the hydrant to render hydraulic transient initiation straightforward. This apparatus can manipulate the network's flow regime while also ensuring easily controlled atmospheric discharge of water, flexible operation and an accurately 
determined valve opening/closure curve, which is essential for the computer model.

\subsubsection{Leak Representation}

Leakage is introduced into the computer model by placing a fixed demand $(0.1,0.5,1$ and $2 \mathrm{~L} / \mathrm{s}$, depending on the scenario) at node J-26 (the only leak location in these early computer runs). Typically, water flow rates from leaks in distribution pipes vary widely, depending on the type and size of leaks (e.g. service connection, joint or corrosion hole leaks vs. circumferential pipe break) and on pipe characteristics (e.g. fluid pressure and pipe material type). Thus, the implementation of several leak flow rates serves here as a sensitivity analysis. It should be noted that the commonly accepted representation for leaks is an orifice function of the form $Q_{l}=C\left(h_{l}\right)^{\alpha}$. This captures the pressure-dependent nature of leaks and its incorporation in a computer model could entail a relevant impact on transient-based leak detection since leaks behave as pressure relief valves, partly attenuating the pressure surge. The inclusion of pressure-dependent leak characterization is planned for a subsequent phase of the study.

\subsubsection{Assessing System Response}

In the computer model, transient pressure signals were recorded at three locations for all scenarios: nodes J-26, J-8130 and J-22 (also indicated in Figure 23.2). Comparisons are made between the pressure time series at these nodes for no-leak and leak scenarios, with the transient always being simulated at the fire hydrant at node J-44 in the manner described. Transient modeling of the system was undertaken using a program called TransAM, developed by HydraTek and Associates Inc.

\subsection{Simulation Results}

\subsubsection{Transient Severity and Pressure Response}

The pressure constraints physically imposed by the pipes' pressure ratings and/or by the utility guidelines) limit the severity of an induced transient. This restriction can have implications for the efficacy of inverse transient analysis aimed at leak detection. The connection is that stronger transients can reveal greater discrepancies between the pressure traces of transient 
responses at the selected nodes, with and without leaks, more clearly, thus exposing any existing leaks or other singularities. An important issue asked in the current study is to what extent the success of inverse transient analysis might depend on the severity of the induced transient.

Figures 23.2 and 23.3 summarize system response for $40 \mathrm{~s}$ opening/ closing $(20 \mathrm{~s}$ each) of the hydrant at J-44 for $50 \mathrm{~L} / \mathrm{s}$ and $85 \mathrm{~L} / \mathrm{s}$ discharge, respectively. Clearly, the larger flow, corresponding to Figure 23.3, leads to a larger pressure fluctuation, and thus to larger stresses on the system. The pressure limits mandated by the utility translate into a maximum discharge from the hydrant of $50 \mathrm{~L} / \mathrm{s}$ with an opening/closing cycle of $40 \mathrm{~s}$.

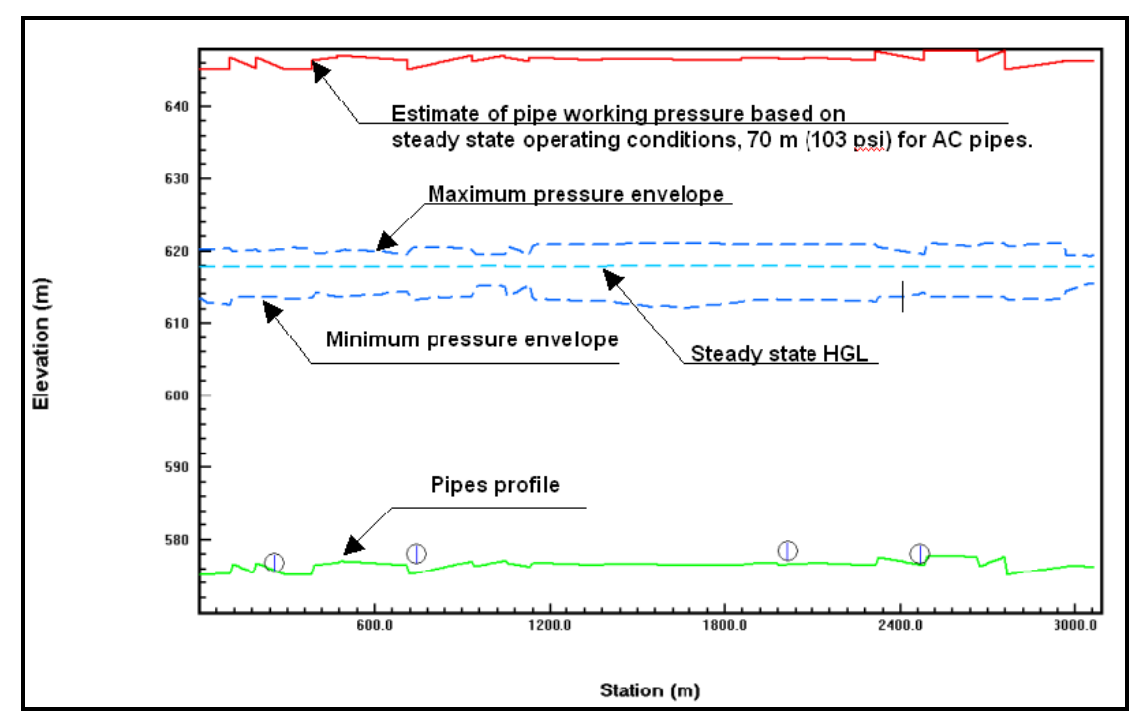

Figure 23.2 Transient due to hydrant $20 \mathrm{~s}$ opening and $20 \mathrm{~s}$ closing; maximum discharge of $50 \mathrm{~L} / \mathrm{s}$. 


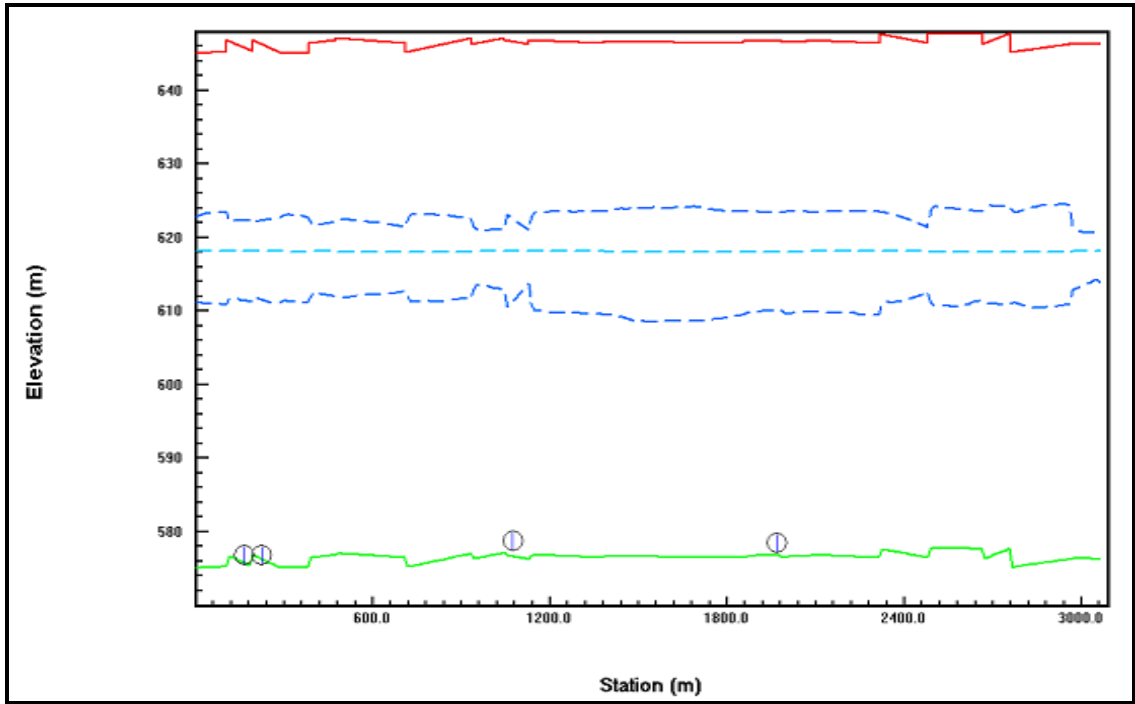

Figure 23.3 Transient due to hydrant 20 s opening and $20 \mathrm{~s}$ closing; maximum discharge of $50 \mathrm{~L} / \mathrm{s}$ (Legend as in Figure 23.2).

Results in Figure 23.4 demonstrate how the severity of a transient induced at the hydrant affects the pressure response at one node in the system (J-8130) in the presence of a $0.5 \mathrm{~L} / \mathrm{s}$ leak at node J-26. Transient severity is governed, in this case, by the duration of the valve cycle time (the time for opening and closing the valve). The pressure signals representing valve cycle times of ten, twenty and thirty seconds correspond with higher, moderate and lower severity transients, respectively. This cycle was defined by ascribing realistic orifice properties to a fire hydrant and by then modulating this "hydraulic size" parameter between a fully open and fully closed position (using a linear change in effective gate closure over the valve duration). As the cycle time drops, the transient is more severe and its impact at the node is reflected by a pressure history exhibiting wider fluctuation and more extreme values. For the same node, Figure 23.5 juxtaposes the series of pressure differences (between the $0.5 \mathrm{~L} / \mathrm{s}$ leak and no-leak scenarios).

An immediate and notable observation is that, for each cycle time, these series behave quite similarly, with almost complete overlap. Conversely, the pressure difference traces in Figure 23.6 exhibit discrepancy at node J-8130 for valve cycle times of 3 and $5 \mathrm{~s}$. This suggests that, at least in this modeled system, leak detection capability via inverse transient analysis is not a 
function of the severity of transient forces above a certain threshold which lies somewhere near a $5 \mathrm{~s}$ cycle time. The judgment call associated with this statement arises from the nature of noise in a real system; almost invariably, real systems have background noise which induces a plus or minus $1 \mathrm{~m}$ pressure variation, unless extreme (and seldom practical) measures are imposed. Thus, even if traces differ in detail, if they are qualitatively closely similar, the ability to extract the influence of a leak rapidly decreases, as shown in Figure 23.5. Moreover, variations in real system attributes from those assumed in the model only highlight these challenges. Apparently then, inverse leak detection can expose small leaks if the network experiences a sufficiently strong transient. However, this may not be possible in practice, depending on pressure restrictions imposed by the owner of the pipe network. The problem facing the proposed field tests is that inducing such transients is prohibited due to the City's pressure restrictions. Thus, another way of revealing leaks with inverse transient analysis must be explored.

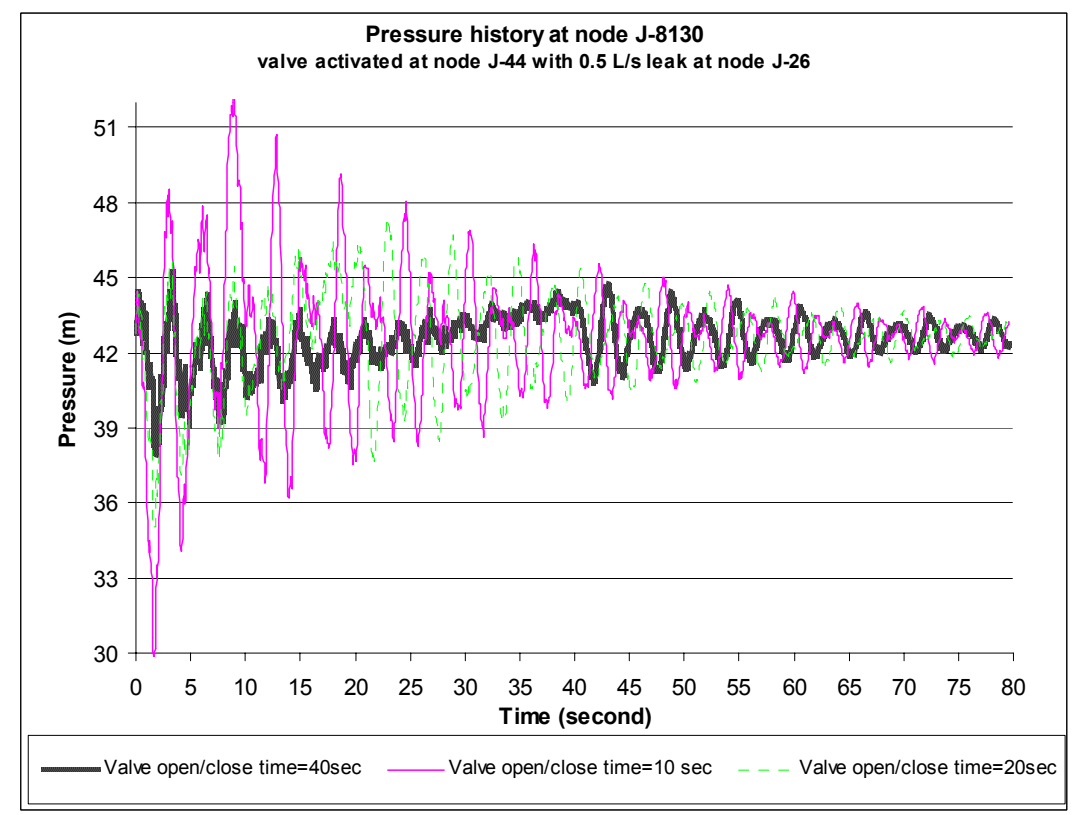

Figure 23.4 Pressure history at node J-8130 for different transient forces resulting from different hydrant ( $\mathrm{J}-44)$ cycle times $(40,20,10$ seconds). 


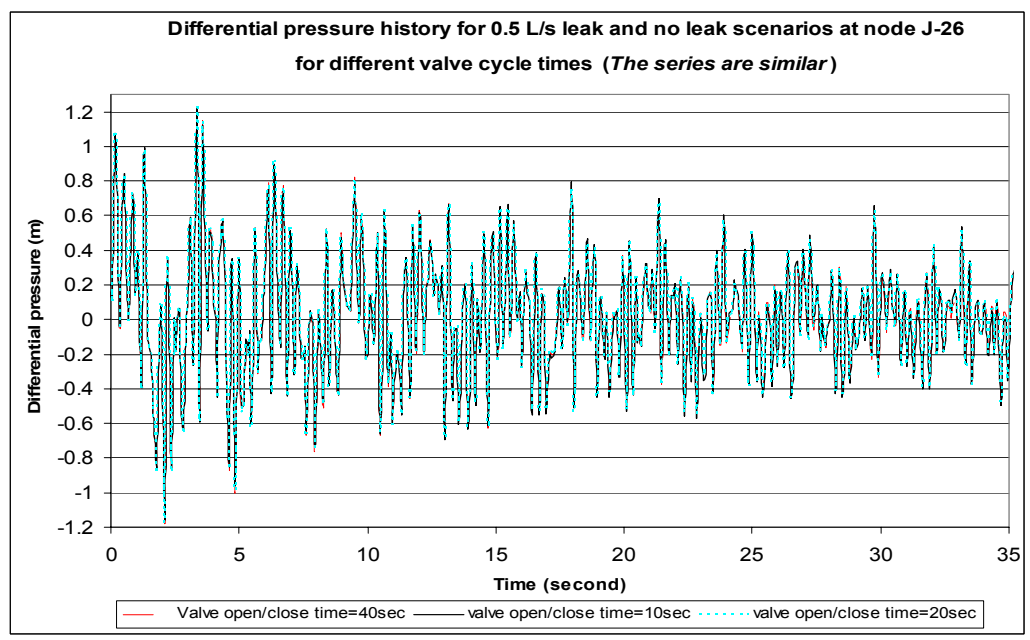

Figure 23.5 Pressure differential series between leak $(0.5 \mathrm{~L} / \mathrm{s})$ and no-leak scenarios for three valve cycle times. The series are similar, suggesting that ITA depends only weakly on transient intensity.

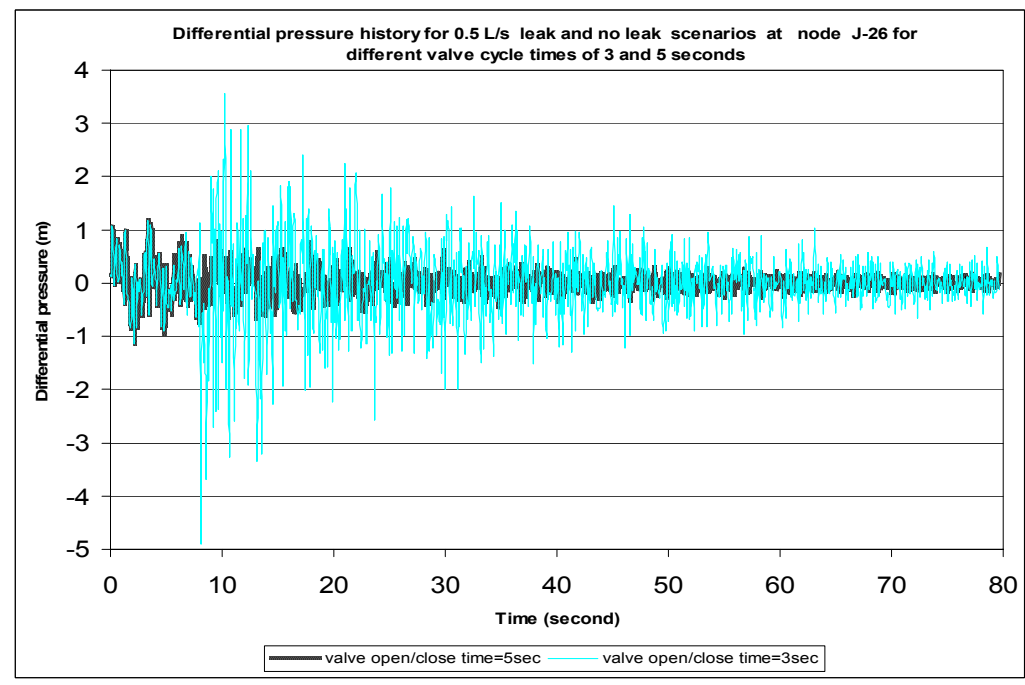

Figure 23.6 Pressure differentials for valve cycle time of 3 and $5 \mathrm{~s}$. This plot suggests that for a cycle time less than $5 \mathrm{~s}$, leak detection is a function of transient intensity. 


\subsubsection{Leakage Size and Detection vs. Transient Severity}

This section examines the role of leak size or, more precisely, discharge rate through the leak at node J-26 and seeks to answer the question: how much does inverse transient leak detection depends on leak size? In finding an answer, four leak discharge rates $(0.1,0.5,1$ and $2 \mathrm{~L} / \mathrm{s})$ were applied in what is essentially a sensitivity analysis. To develop an idea of system response in the absence of leakage and to establish a baseline for comparison, a nonleaky system was first scrutinized. Figure 23.2 indicates the minimum/maximum pressure envelopes for an induced transient event resulting from $50 \mathrm{~L} / \mathrm{s}$ peak discharge at the hydrant for a 20 -second valve cycle time. Figures 23.7 and 23.8 depict the pressure signal observed at nodes $\mathrm{J}-44$ and node $\mathrm{J}-8130$, respectively.

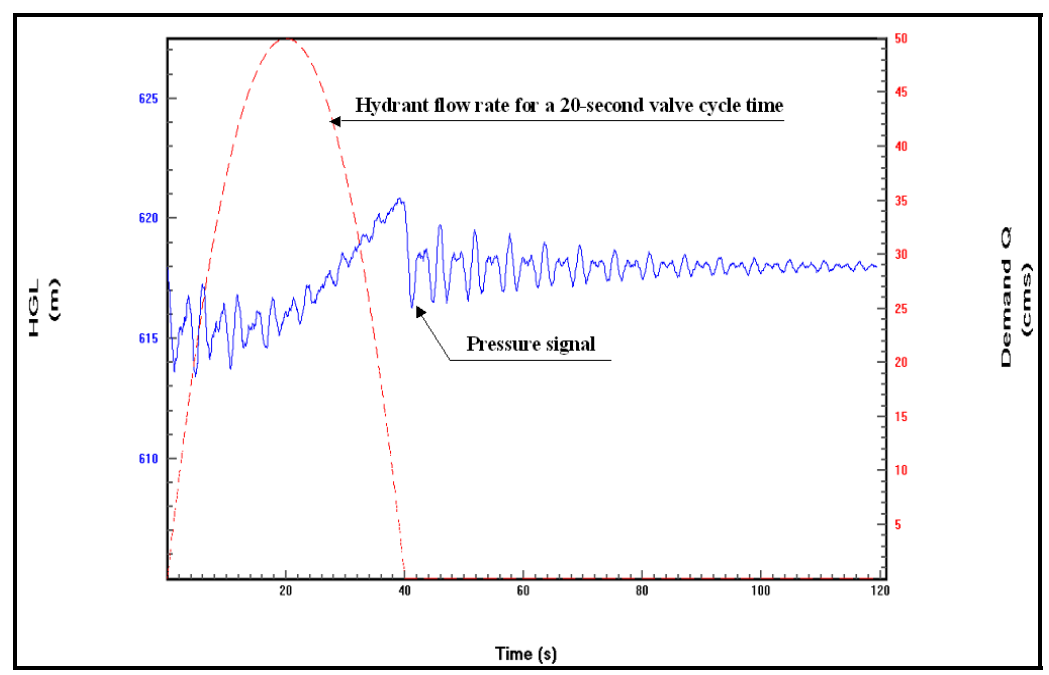

Figure 23.7 Pressure head and hydrant flow rate at node J-44.

The pressure traces at $\mathrm{J}-8130$, as a result of the same induced transient described for the no-leak scenario, for varying levels of discharge $(0.1,0.5$, 1 , and $2 \mathrm{~L} / \mathrm{s}$ ) were found to be almost similar. This similarity underscores the importance of re-running a steady state simulation for each new leak level prior to conducting a transient analysis, for the purpose of avoiding erroneously similar results. 


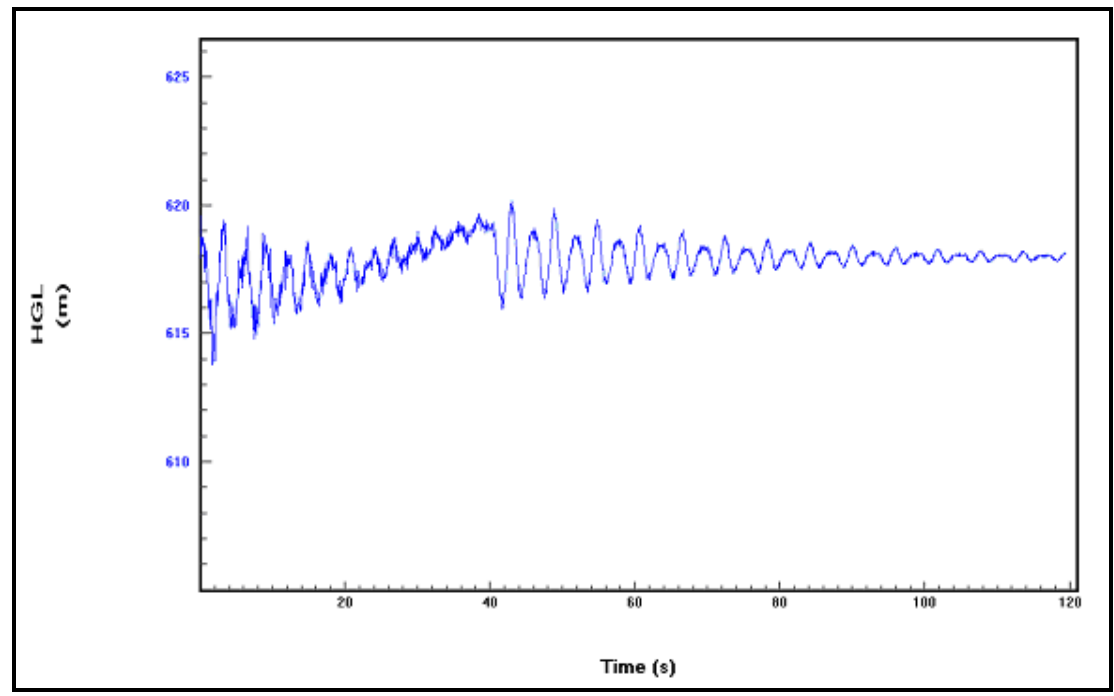

Figure 23.8 Pressure head at node J-8130.

\subsubsection{Comparing Pressure Variations for Different Scenarios}

Comparison of pressure responses at node J-8130 for all scenarios (i.e., different leak sizes at node J- 26 with different severity of transients induced at node J-44) serves to emphasize the relationships between transient behaviour and leak size. Progressing from Figure 23.9 to 23.11, it becomes clear that the method's success in exposing a leak escalates as the leak size increases. The larger range of pressures associated with the presence of a leak can be attributed to a larger flow of water, and thus kinetic energy, through the system, facilitating the detection of the leak. Figure 23.12 superimposes the series of pressure differentials (leak and no-leak) for all four leak discharges on the same set of axes. It illustrates the relationship developed in Figures 23.9-23.11; that is, smaller leaks exhibit tighter pressure variations and narrower differentials than do larger leaks, suggesting that inverse transient analysis is more effective at exposing larger leaks. Thus, if the technique is less well suited to revealing small leaks, its applicability in real systems for which ongoing water loss is largely owing to the presence of many distributed small leaks is questionable. In practice, however, water flow rates from leaks in distribution pipes vary widely, and 
acoustic leak surveys may fail to detect "quiet" large leaks. An example of this problem for a ten litres per second leak was reported by Hunaidi and Brothers (2007). Therefore, application of inverse transient analysis in tandem with periodic acoustic surveys may provide a more efficient and economic leakage management strategy. Inverse transient analysis can also be implemented in conjunction with a DMA-based leakage management strategy to help narrow down the location of leaks within large DMAs.

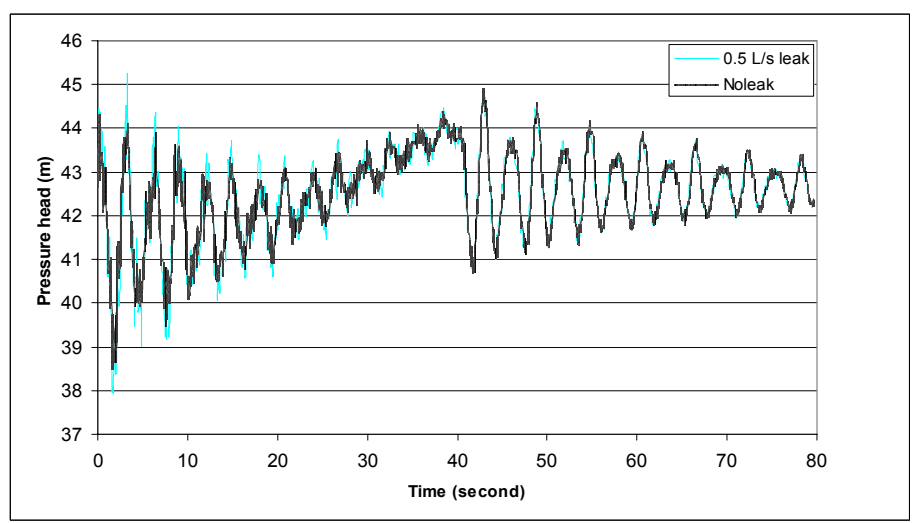

Figure 23.9 Pressure head variations at node J-8130, for no-leak and $0.5 \mathrm{~L} / \mathrm{s}$ scenarios.

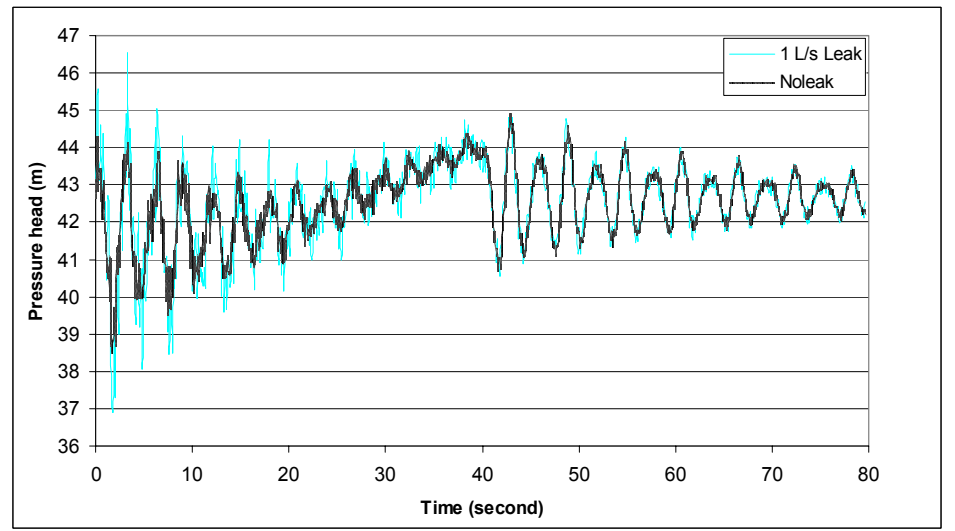

Figure 23.10 Pressure head variations at node J-8130, for no-leak and $1.0 \mathrm{~L} / \mathrm{s}$ scenarios. 


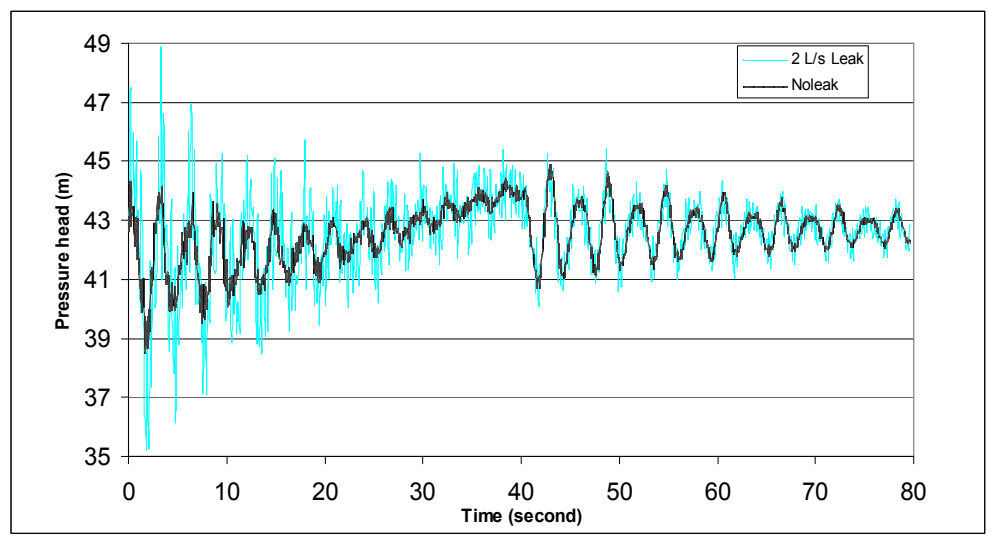

Figure 23.11 Pressure head variations at node J-8130, for no-leak and $2.0 \mathrm{~L} / \mathrm{s}$ scenarios.

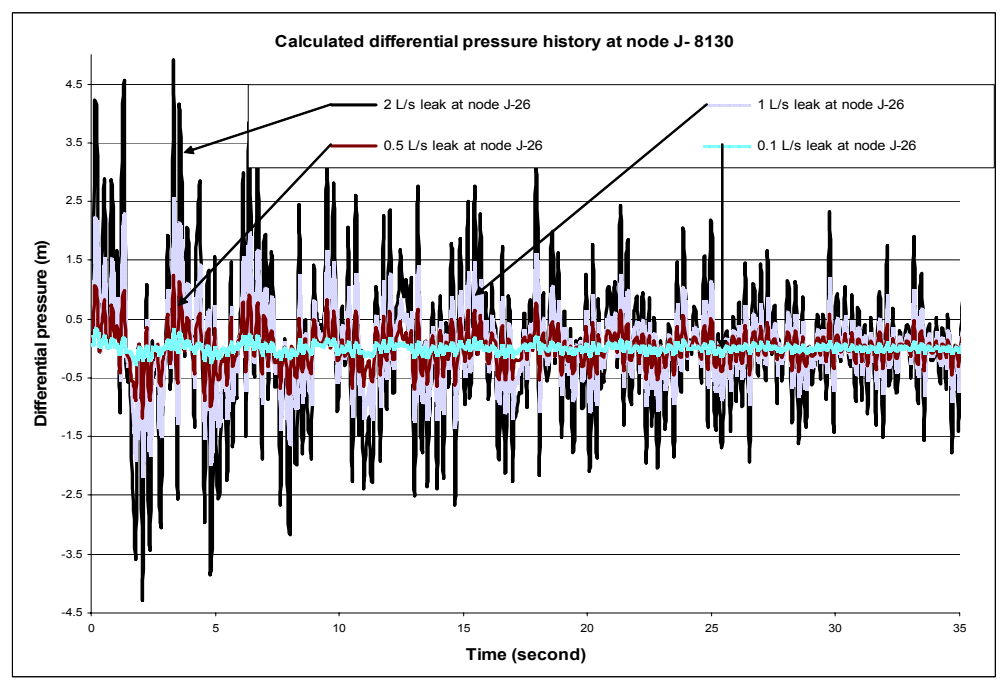

Figure 23.12 Pressure difference history at node J-8130 with series corresponding to different leak sizes juxtaposed on the same set of axes. The differentials for leakage below $0.5 \mathrm{~L} / \mathrm{s}$ are very small, rendering small leak $(<0.5$ $\mathrm{L} / \mathrm{s}$ ) detection difficult. While they could be enlarged with more severe transients, pressure variations would be steep and risk transgressing safe limits. Thus, for small leak detection and weaker transients, tests must be conducted with a minimum of noise in the system. 


\subsection{Conclusion}

Numerous studies have demonstrated the feasibility of the ITA method to detect leaks. However, the effectiveness and accuracy of the method relies on the availability of a reliable and calibrated transient model of the system. Combining the ITA method with other approaches that are not dependent on the transient model (e.g. the frequency domain analysis of the system's response, the analysis of reflected transient signals) would render the method more practical and significantly improve its chance of success as a feasible leak detection tool.

Based on the results of the simulation experiment, a tentative conclusion could be made that the inverse transient analysis method may be an effective tool for detecting leaks of intermediate to large size. Generally, the ITA method is more effective at detecting large leaks (or breaks) and may not be able to detect small leaks, within the acceptable levels of induced transient pressures. However, the ITA method can be used in conjunction with other leak detection methods to increase their efficiency and economic viability. For example, the method can serve as a supplement to DMAs or acoustic surveys by helping narrow down the location of leaks within large DMAs.

Field-testing of the ITA method should be conducted under carefully controlled conditions to mitigate the risks that may arise due to severe transients. A systematic procedure for field implementation that addresses risk concerns still needs to be developed. Also, further work is planned to develop practical guidelines for implementing the method in the field (e.g. optimum size of test areas, number and placement of pressure transducers, appropriate transient levels, etc.).

\section{Acknowledgment}

This work is a collaborative effort between the National Research Council of Canada (NRC), the University of Toronto, HydraTek and Associates Inc., and the City of Regina. The writers thank Dr. David Hubble and the anonymous reviewers for their valuable suggestions and feedback.

\section{References}

Colombo, A.F, Lee, P. and Karney, B.W. (2008). "A selective literature review of transient based leak detection methods." Submitted to the Journal of HydroEnvironment Research. 
Covas, D., Stoianov, I., Butler, D., Maksimovic, C., Graham, N. and Ramos, H. (2001). "Leakage detection in pipeline systems by inverse transient analysis - from theory to practice." Proc. Sixth Int. Conference on Computing and Control in the Water Industry (CCWI), Leicester, England, September 2001.

Fantozzi, M., Di Chirico, G., Fontana, E., and Tonolini, F. (1993). Leak inspection on water pipelines by acoustic emission with cross-correlation method. AWWA Annual Conference, San Antonio, Texas, USA, June 6-10, 1993.

Fuchs, H. and Riehle, R. (1991). Ten years of experience with leak detection by acoustic signal analysis. Applied acoustics, 33(1), Elsevier Science Publishers Ltd., England (1990).

Hunaidi, O., Chu, W., Wang, A., and Guan, W. (2000). Detecting Leaks in Plastic Pipes, Journal AWWA, 92 (2), pp. 82-94, February 2000, Also posted at: "irc.nrc.gc.ca/fulltext/nrcc43058.pdf"

Hunaidi, O. (2005). "Economic comparison of periodic acoustic surveys and DMA-based leakage management strategies," Leakage 2005 Conference Proceedings (Halifax, N.S. September 12, 2005), pp. 322-336, September 01, 2005. Also available at http://irc.nrc-cnrc.gc.ca/pubs/fulltext/nrcc48196/nrcc48196.pdf

Hunaidi, O.; Wang, A.; Bracken, M.; Gambino, T.; Fricke, C. (2004). Acoustic methods for locating leaks in municipal water pipe networks, International Conference on Water Demand Management, Dead Sea, Jordan, May 30-June 3,2004. Also available at http://irc.nrc-cnrc.gc.ca/pubs/fulltext/nrcc47062/nrcc47062.pdf

Hunaidi, O., and Brothers, K. (2007). "Night Flow Analysis of Pilot DMAs in Ottawa." Water Loss Specialist Conference, International Water Association, pp. 32-46, Bucharest, Romania, 23-26 September 2007. Also available at http://irc.nrccnrc.gc.ca/pubs/fulltext/nrcc50299/nrcc50299.pdf

IWA (2007) District Metered Areas Guidance Notes. Water Loss Task Force, Efficient Operation and Management Specialist Group, International Water Association.

Liggett, J.A. and Chen, L-C. (1994). "Inverse transient analysis in pipe networks." Journal of Hydraulic Engineering, ASCE, 120(8), 934-955.

Pudar, R.S. and Liggett, J.A. (1992). "Leaks in pipe networks.” Journal of Hydraulic Engineering, ASCE, 118(7), 1031-1046.

Saldarriaga, J.G., Araque Fuentes, D.A., Castañeda Galvis, L.F. (2006). "Implementation of the hydraulic transient and steady oscillatory flow with genetic algorithms for leakage detection in real water distribution networks." Eighth Annual Water Distribution Systems Analysis Symposium, Cincinnati, OH, August 27-30, 2006.

Tang, K.W., Karney, B.W. and Brunone, B. (2001). Leak detection using inverse transient calibration and GA - some early successes and future challenges. Proc. Sixth Int. Conference on Computing and Control in the Water Industry (CCWI), Leicester, England, September 2001.

Vitkovský, J.P., Simpson, A.R., and Lambert, M.F. (2000). "Leak detection and calibration of water distribution systems using transients and genetic algorithms." Journal of Water Resources Planning and Management, ASCE, 126(4), 262-265. 

